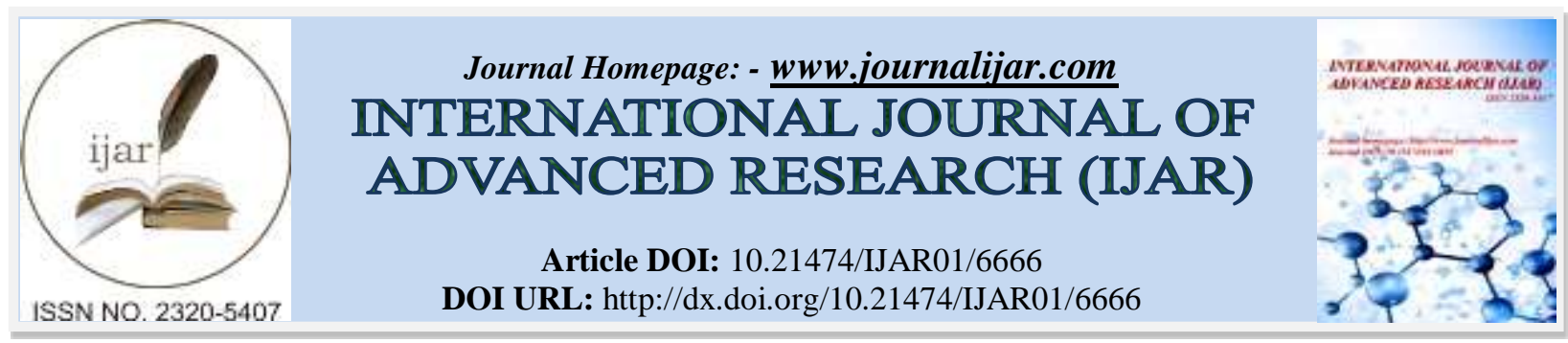

RESEARCH ARTICLE

\title{
RADIOLOGICAL ASSESSMENT OF HEALING IN PERIAPICAL DEFECTS TREATED WITH PLATELET RICH PLASMA AND PLATELET RICH FIBRIN ALONG WITH ALLOPLASTIC MATERIAL- A COMPARATIVE STUDY.
}

\section{Dr. Naga Malleswara Rao. $\mathbf{I}^{1}$, Dr. poornima Sowjanya ${ }^{2}$, Dr. N V V Satya Bhushan ${ }^{3}$ and Dr. Venkata Rajesh Kumar Darsi ${ }^{4}$.}

1. MDS, Assistant Professor, Dept of Oral and Maxillofacial Surgery.

2. MDS, Assistant Professor, Dept of Oral and Maxillofacial Surgery, KIMS Dental College and Hospital, Amalapuram.

3. MDS, Professor and H.O.D, Dept of Oral and Maxillofacial Surgery, GITAM Dental College and Hospital, Vishakhapatnam.

4. MDS, Assistant Professor, Dept of Community Dentistry, KIMS Dental College and Hospital, Amalapuram.

\section{Manuscript Info}

Manuscript History

Received: 04 January 2018

Final Accepted: 06 February 2018

Published: March 2018

Keywords:-

platelet rich plasma, platelet rich fibrin,

growth factors, regeneration.

\section{Abstract}

Platelet rich plasma and platelet rich fibrin are the two platelet concentrates that are introduced in to the field of medicine for application in various specialities. In oral and maxillofacial surgery they are currently used for bone regenerative procedures. Both the platelet concentrates, due to the presence of growth factors in the platelets helps to fasten the healing process. Our aim is to assess the healing in periapical osseous defects treated with both PRP and PRF.

Copy Right, IJAR, 2018,. All rights reserved.

\section{Introduction:-}

Osseous regeneration in the periapical defects as a result of trauma or pathology in oral and maxillofacial region has been a challenge. Generally, it takes 6-12 months to complete the osseous regeneration in the defect after peri apical surgery, so different bio materials are used to fasten the same. The use of autogenous bone has remained the gold standard in restoring bone defects, but there are some limitations like limited availability, second surgical site, prolonged operative period and donor site morbidity. ${ }^{1}$ To overcome these difficulties alloplastic bone substitutes have come into the picture. The most commonly used is synthetic hydroxyapatite due to its chemical composition being similar to that of human bone, non- toxic nature, high chemical stability with less inflammation and antigenic reactions ${ }^{2}$.

The next step in osseous regeneration is tissue engineering which was achieved by introduction of platelet-rich concentrates, by utilizing the inherent property of platelets to release various growth factors and thereby quickening the healing process. One such innovation in dentistry is the preparation and use of platelet rich plasma (PRP), which is a concentration of platelets and growth factors released from platelets ${ }^{\mathbf{3}}$.

Recently, a new platelet concentrate was introduced in France in the year 1978 by Choukroun called Platelet- rich Fibrin (PRF).It is the second generation platelet concentrate. It has the advantage of natural way of activation, prolonged release of growth factors, slow polymerization and simplified production protocols. ${ }^{4}$ 
Following the dictum that "if a few are good, then a lot may be better", it seems very logical that increasing the concentration of platelets at the osseous defect will increase the concentration of growth factors, which may lead to more rapid osseous regeneration. This has encouraged us to radiographically compare the efficacy of the platelet- rich plasma and platelet rich fibrin mixed with hydroxyapatite in the healing of periapical defects.

\section{Materials and methods:-}

This study was conducted in the department of Oral and Maxillofacial Surgery, GITAM dental college and hospital, Visakhapatnam. 60 patients who reported to the Department of Oral and Maxillofacial Surgery presenting with the Periapical defects were randomly selected for the study after obtaining Ethical committee approval. They are divided in to two groups, 30 in each group. Patients suffering from any systemic disorders, blood dyscrasiasis, patients on antiplatelet / anticoagulant therapy, patients with history of recent myocardial infarction and pregnant women were excluded from the study.

In this study, all the lesions were diagnosed as radicular cysts except for one case which was diagnosed dentigerous cyst. The offending teeth were saved conservatively by performing root canal therapy while in very few patients offending tooth was extracted due to poor prognosis. The surgical procedure included enucleation of the cystic lining and placement of platelet rich plasma or platelet rich fibrin mixed with hydroxyapatite bone grafting material. Platelet rich plasma and platelet rich fibrin were prepared according to the standard protocols ${ }^{5}$.

\section{Surgical procedure:-}

Local anesthesia using 2\% lignocaine with 1:80000 adrenaline was given in the area of surgical interest under aseptic conditions. Full thickness mucoperiosteal flap was raised from the buccal or palatal side and the defect was exposed from all the sides (FIG 1\&8). The defect was curetted and the cystic lining was removed. Wound is irrigated with saline and all the foreign particles were removed from the defect. The wound was dried with sterile gauze piece to achieve proper hemostasis. Once the wound is dried, PRP or PRF is placed on to the walls and base of the defect and the remaining cavity was totally filled with hydroxyapatite material (FIG 2\&9). Overfilling and under filling is avoided, as it precludes proper flap closure. The wound was closed primarily with interrupted sutures using 3-0 black silk. Postoperative antibiotics and analgesics were prescribed.

The patients were recalled on the next day of surgery for check-up. The patients were recalled after 7 days for suture removal and thereafter at the end of 1, 3 and 6 months. Intra oral periapical radiographs were taken and assessed for bone regeneration (FIG 3-7 \& 10-13).

\section{Results:-}

60 patients were included in the study who were divided randomly in to PRP group and PRF group. Intraoral periapical radiographs were taken at 1,3 and 6 months interval to assess the blending of the bone margins and formation of trabecular bone. Both PRP and PRF groups did not show any changes except for the resorption of the graft material in the radiograph taken after 1 month. After 3 months all the cases in PRP group showed blending of the margins where as only 24 cases showed blending of margins in PRF group. After 6 months margins were completely blended in both the groups. Trabecular bone formation is not seen after 1 month in any of the groups. After 3 months, 6 cases showed trabecular bone formation in PRP group where as 15 cases showed trabecular bone formation in PRF group. Both the groups showed complete bone formation after 6 months. Chi Square test was used to compare the observations between both the groups. P value of $<0.05$ is statistically significant.

\section{Blending Of Bone Margins}

\begin{tabular}{|c|c|c|c|c|c|}
\hline 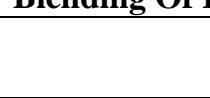 & & & & $\begin{array}{l}\text { CHISQUARE } \\
\text { TEST }\end{array}$ & P VALUE \\
\hline \multirow[b]{4}{*}{$1^{\mathrm{ST}}$ MONTH } & \multirow[b]{2}{*}{ PRP } & NEGATIVE & $30(100 \%)$ & \multirow[b]{4}{*}{ - } & \multirow[b]{4}{*}{-} \\
\hline & & POSITIVE & 0 & & \\
\hline & \multirow[b]{2}{*}{ PRF } & NEGATIVE & $30(100 \%)$ & & \\
\hline & & POSITIVE & 0 & & \\
\hline \multirow[b]{4}{*}{$3^{\mathrm{RD}}$ MONTH } & \multirow[b]{2}{*}{ PRP } & NEGATIVE & 0 & \multirow{4}{*}{6.667} & \multirow[b]{4}{*}{ 0.010* } \\
\hline & & POSITIVE & $30(100 \%)$ & & \\
\hline & \multirow[b]{2}{*}{ PRF } & NEGATIVE & $6(20 \%)$ & & \\
\hline & & POSITIVE & $24(80 \%)$ & & \\
\hline
\end{tabular}




\begin{tabular}{|l|l|l|l|l|}
\hline & \multirow{3}{*}{ PRP } & NEGATIVE & 0 & \multirow{3}{*}{} \\
\cline { 2 - 4 } $\mathbf{6}^{\text {TH }}$ MONTH & \multirow{2}{*}{ PRF } & POSITIVE & $30(100 \%)$ \\
\cline { 3 - 4 } & NEGATIVE & 0 & \\
\cline { 2 - 4 } & POSITIVE & $30(100 \%)$ & - \\
\hline
\end{tabular}

Table 1:- showing statistically significant difference in blending of margins between the groups at $3^{\text {rd }}$ month followup.

Trabecular Bone Formation

\begin{tabular}{|c|c|c|c|c|c|}
\hline & & & & $\begin{array}{l}\text { CHISQUARE } \\
\text { TEST }\end{array}$ & P VALUE \\
\hline \multirow[b]{4}{*}{$1^{\mathrm{ST}}$ MONTH } & \multirow[b]{2}{*}{ PRP } & NEGATIVE & $30(100 \%)$ & \multirow[b]{4}{*}{-} & \multirow[b]{4}{*}{-} \\
\hline & & POSITIVE & 0 & & \\
\hline & \multirow[b]{2}{*}{ PRF } & NEGATIVE & $30(100 \%)$ & & \\
\hline & & POSITIVE & 0 & & \\
\hline \multirow[b]{4}{*}{$3^{\mathrm{RD}}$ MONTH } & \multirow[b]{2}{*}{ PRP } & NEGATIVE & $24(80 \%)$ & \multirow[b]{4}{*}{5.934} & \multirow[b]{4}{*}{$0.015 *$} \\
\hline & & POSITIVE & $6(20 \%)$ & & \\
\hline & \multirow[b]{2}{*}{ PRF } & NEGATIVE & $15(50 \%)$ & & \\
\hline & & POSITIVE & $15(50 \%)$ & & \\
\hline \multirow[b]{4}{*}{$6^{\mathrm{TH}}$ MONTH } & \multirow[b]{2}{*}{ PRP } & NEGATIVE & 0 & \multirow[b]{4}{*}{ - } & \multirow[b]{4}{*}{-} \\
\hline & & POSITIVE & $30(100 \%)$ & & \\
\hline & \multirow[b]{2}{*}{ PRF } & NEGATIVE & 0 & & \\
\hline & & POSITIVE & $30(100 \%)$ & & \\
\hline
\end{tabular}

Table 2:- showing statistically significant difference in trabecular bone formation between the groups at $3^{\text {rd }}$ month follow up.

* denotes p value $<0.05$ - statistically significant.

\section{Discussion:-}

In recent years, the process of bone regeneration in periapical defects has been greatly enhanced by the identification of growth factors in platelets. Platelet concentrates prepared from patient's own blood is something that is new and the strategy is to accelerate the initiation of bone regeneration by enhancing the effect of growth factors present in platelets ${ }^{6}$. Two platelet concentrates, platelet rich plasma (PRP) and platelet rich fibrin (PRF) were introduced in to the field of Oral and Maxillofacial Surgery for enhancing bone regeneration. Growth factors like platelet derived growth factor (PDGF-AB), transforming growth factor $\beta$ (TGF $\beta$ ), insulin like growth factor (IGF), epidermal growth factor (EGF) are released from platelet $\alpha$ granules. It is accepted that the transcription core binding factor alpha 1 (cbfa 1 mRNA) is a critical regulator of osteoblast differentiation.

Application of platelets present in PRP has begun with the study of Marx et $\mathbf{a l}^{7}$ in 1998. Their data strongly suggested that adding PRP to bone grafts accelerated the rate and degree of bone formation. With the addition of autologous PRP the local availability of the growth factors at the site of osseous defects is enhanced by about three fold or greater. Later second generation platelet concentrate, platelet rich fibrin (PRF) was introduced by choukron et al. The PRF production protocol is very simple. It has the characteristic of polymerizing naturally and slowly during centrifugation. This slow polymerization mode confers a particularly favorable physiologic architecture to support the healing process. ${ }^{4}$

We used this principle for enhancing the osteoconductive property of bone graft by adding PRP, PRF in the regeneration of osseous defects among 60 patients. As there are less comparative clinical studies to evaluate bone regeneration in periapical osseous defects using both PRP and PRF, we compared both the platelet concentrates. Patients are randomly divided in to two groups PRP group, PRF groups with each group containing 30 patients. The standard protocol for preparing PRP and PRF was used. Blending of margins and Trabecular bone formation are the two parameters studied in intraoral perapical radiographs at $1^{\text {st }}$ month, $3^{\text {rd }}$ month, $6^{\text {th }}$ month post operatively and compared between the groups.

In our study, we observed significant amount of blending of margins in all the 30 patients by the end of $3^{\text {rd }}$ month in PRP group, which is statistically significant. This may be due to the initial rapid release of growth factors in PRP due 
to activation by thrombin. Whereas trabecular bone formation was not significant at $3^{\text {rd }}$ month except in 6 cases. These results were attributed to the findings of Kanno et al (2005) ${ }^{\mathbf{8}}$ that PRP also effects the proliferation and differentiation of human osteoblasts. By the end of 6 months blending of margins, trabecular bone formation is seen in all the 30 cases. These results are correlated with studies done by Praveen Reddy B (2010) ${ }^{6}$, Bielecka A C et al $\mathbf{~ ( 2 0 0 8 ) ~}^{9}$, Oyama T et al (2004) ${ }^{\mathbf{1 0}}$, Sammartino $\mathbf{G}$ et al in (2005) ${ }^{\mathbf{1 1}}$. Whereas in 4 cases, trabecular bone that is formed at $6^{\text {th }}$ month was not complete and there was a radiolucent area in the radiograph at the center of the lesion which was similar to the finding of Lacoste et al (2003) ${ }^{\mathbf{1 2}}$ who suggested that the main reason for the inferior effect of PRP on bone regeneration is that high concentration of thrombin used in clinical practices caused fast release of mitogens. This release might have occurred before the initiation of outgrowth of osteoblasts from the surrounding tissue, thus leaving fewer mitogens to be released after cellular growth had started. The effect of PRP on bone regeneration is thus limited.

Standard protocol by Choukroun for preparing $\mathrm{PRF}^{4}$ was used for the study. Handling PRF is easy, as it is fibrin clot and can be easily adapted to the walls of the defect because of its fibrin network. Dohan D M et al (2006) ${ }^{\mathbf{1 3}}$ conducted a study in which platelet cytokine quantification was carried out by ELISA to understand the new biomaterial. The results implies that PRF would be able to progressively release cytokines during fibrin matrix remodelling. In PRF group, only 6 cases showed blending of margins at 3 months. 15 out of 30 cases showed trabecular bone formation by the end of $3^{\text {rd }}$ month. There is statistically significant difference between the two groups in trabecular bone formation. All the cases in PRF group showed complete trabecular bone formation by the end of 6 months. Similar results were obtained by Lee $\mathbf{J} \mathbf{W}$ et al (2012) ${ }^{\mathbf{1 4}}$ in their animal study which showed that more amount of bone regeneration occurred in a peri implant defect filled with PRF than unfilled defects.

There are no complications like postoperative infection in any of our cases. This is due to the immune organizing mode action of platelet concentrates as observed by Dohan D M et al (2006). ${ }^{15}$

PRF when used along with hydroxyapatite not only accelerates hard tissue healing but also increases the density of the bone formed. Choukroun J et al (2006) ${ }^{16}$, Horowitz et al (2009) ${ }^{17}$,Dohan et al (2008) ${ }^{18}$ observed denser bone formation in their studies on sinus augmentation for implant placement. It has been reported by Pradeep A R et al (2012) ${ }^{\mathbf{1 9}}$ that combination of HA with PRF resulted in better results when used in intrabony defects. Inspite of the theoretical advantage of using PRF for osseous regeneration we could find statistically significant difference between PRP and PRF groups.

We found PRP protocol to be more complicated pertaining to the two centrifugation cycles that is needed and separation of the buffy coat from the initial spin. Handling the material along with hydroxyapatite and adapting the material to the osseous defect was difficult because of the gel like consistency of the material. Whereas PRF has relative ease of preparation and better handling properties.

CASE NO-1:- Placement Of Prp, Hydroxyapatite In Periapical Defcet

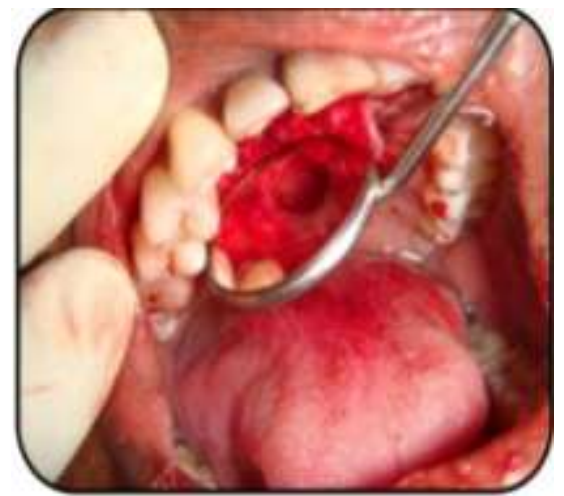

Fig 1:-showing osseous defect i.r.t 12,13

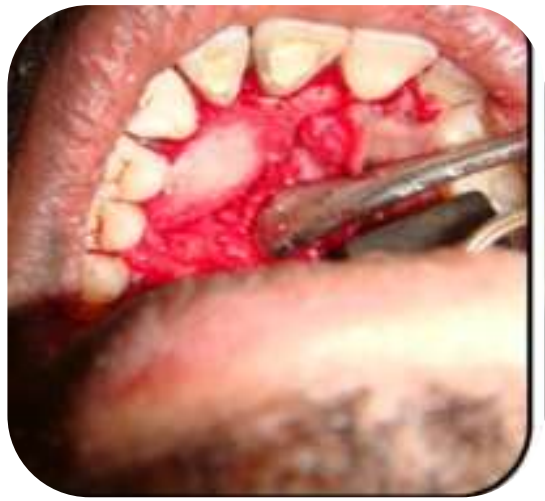

Fig 2:- showing placement of PRP+Hydroxyapatite 


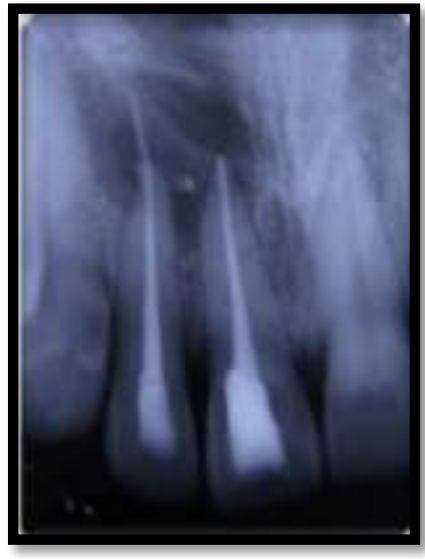

Fig 3:- pre o.p IOPA showing radiolucency i.r.t 11,12

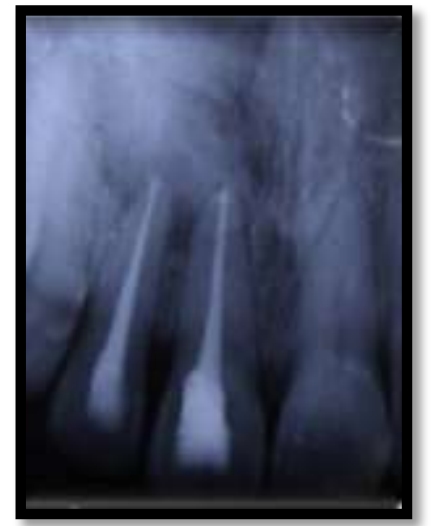

Fig 4:- immediate post o.p IOPA showing graft in place
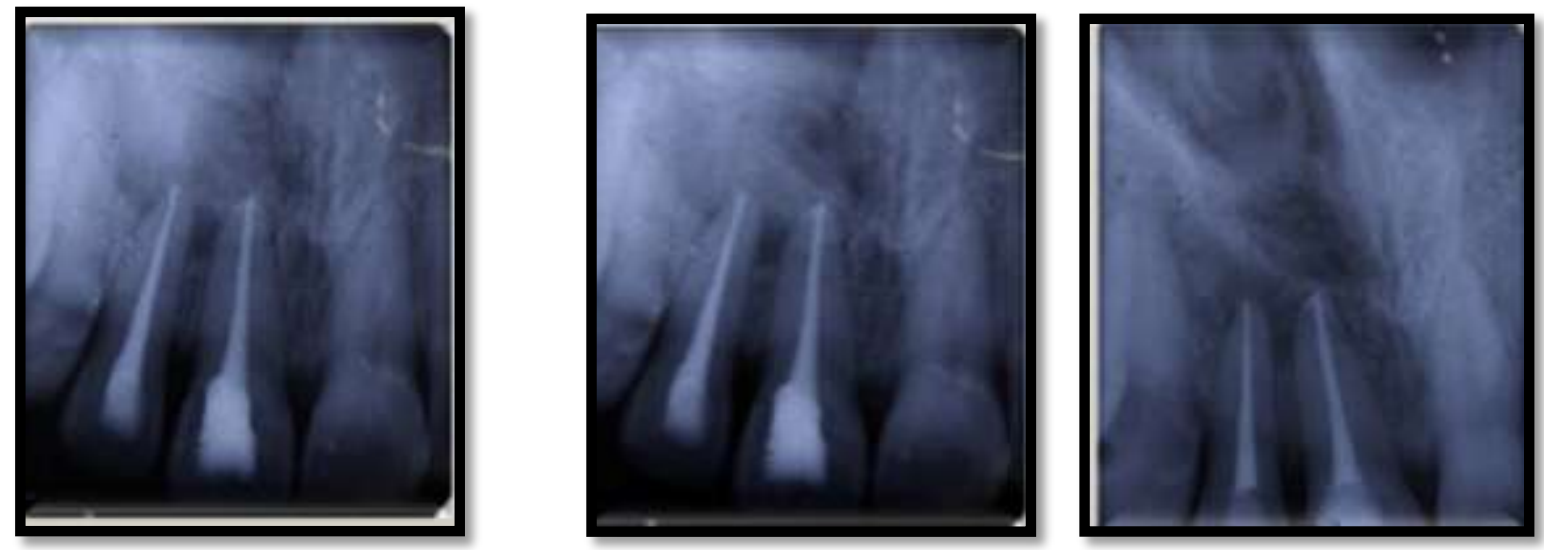

Fig 5:-IOPA of $1^{\text {st }}$ month follow up

Fig 6:-IOPA of $3^{\text {rd }}$ month follow up

Fig 7:- IOPA of $6^{\text {th }}$ month follow up

Case No-2 Placement Of Prf,Hydroxyapatite In Periapical Defcet

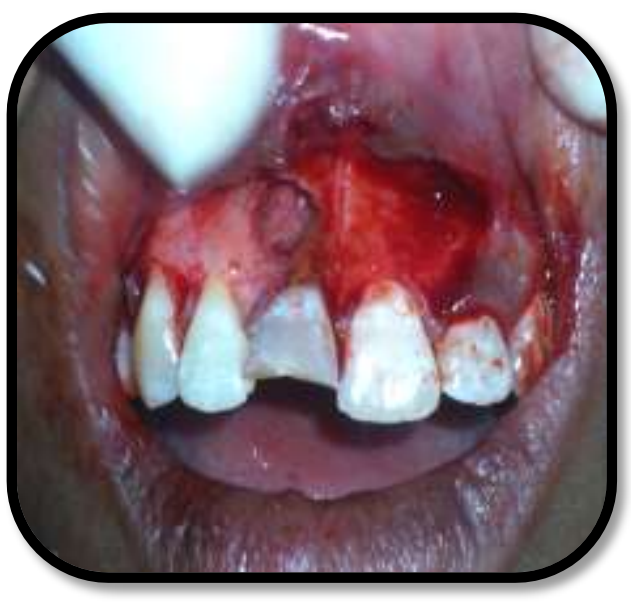

Fig 8:- showing osseous defect i.r.t 11,12

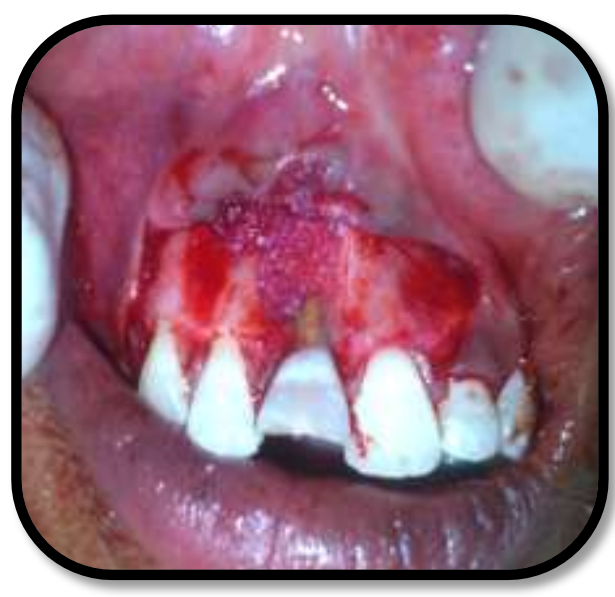

Fig 9:- showing placement of PRF+Hydroxyapatite 


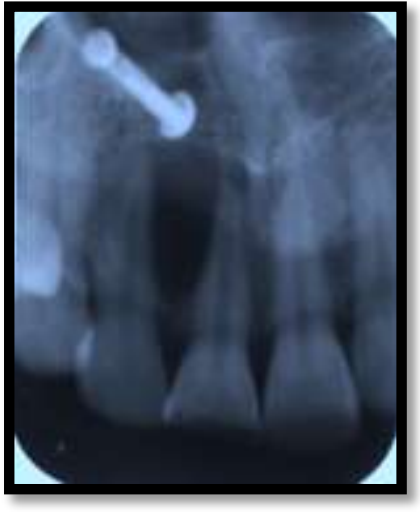

Fig 10:- pre o.p IOPA showing radiolucency i.r.t 11,12

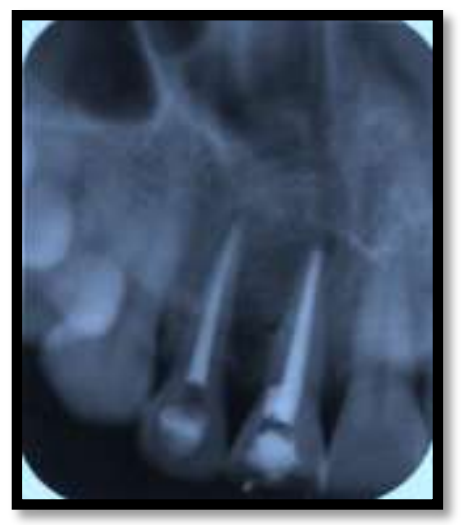

Fig 12:-IOPA of $3^{\text {rd }}$ month follow up

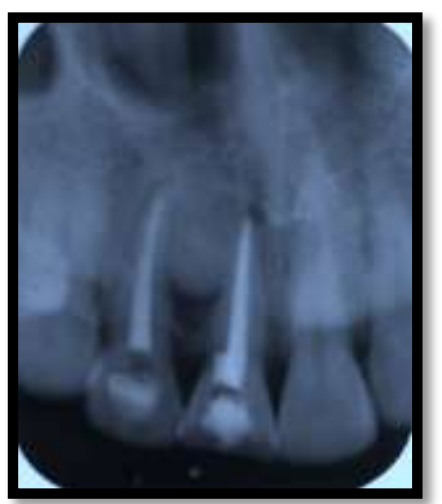

Fig 11:- immediate post o.p IOPA showing graft in place

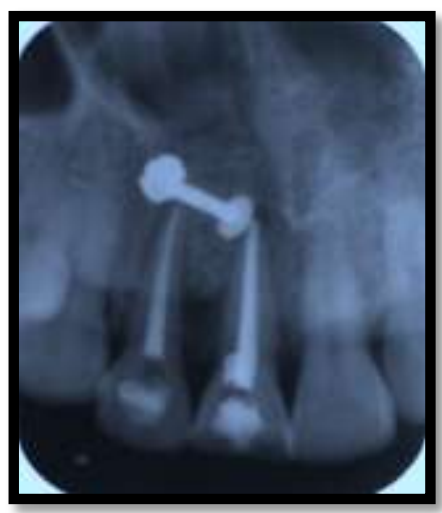

Fig 13:-IOPA of $6^{\text {th }}$ month follow up

\section{Conclusion:-}

Bone regeneration in osseous defects can be accelerated by using recently developed materials like platelet rich plasma and platelet rich fibrin. Currently existing literature suggests the use of both PRP and PRF in osseous defects. There are few studies comparing the efficacy of both the materials in the literature. So, we aimed to compare the efficacy in terms of healing of periapical defects. Though there is a theoretical advantage of using PRF over PRP, we could find statistically significant differences in terms of blending of margins and trabecular bone formation in our cases. Small size of the sample, short follow up period and variability in the size of the defects may be the drawbacks of the study. Future studies are required with large sample size, longer follow up period and defect size should be standardized in order to establish the efficacy of platelet concentrates in the healing of osseous defects.

\section{Bibliography:-}

1. Thorwarth M, Stefan SM, Kessler P,.Bone regeneration in osseous defects using a resorbablenano particular hydroxyapatite. J Oral Maxillofac surg. 2005;63: 1626-1633.

2. Ravi Shankar, Deepak Singh, Ravi Jain. Bone regeneration in osseous defects using hydroxyapatite graft and the extent of ossification in osseous defects treated without grafts: A comparative evaluation. J Maxillofac oral surg. 2011; 10(2): 123-126.

3. Shobha P, Aditi T. Platelet Concentrates: Past, Present and Future. J. Maxillofac. Oral Surg. 2011; 10(1):45-49.

4. Dohan D M, Choukroun J, Diss A, Dohan S L. Platelet-rich fibrin (PRF): A second-generation platelet concentrate.Part I: Technological concepts and evolution. Oral Surg Oral Med Oral Pathol Oral RadiolEndod. 2006; 101: E37-44.

5. Schlege KA, Zimmermann R, Thorwarth M, Sinus floor elevation using autogenous bone or bone substitute combined with platelet-rich plasma. Oral Surg Oral Med Oral Pathol Oral RadiolEndod. 2007; 104: e15-e25.

6. Reddy P, Nagaveni, Shankar U, Efficacy of PRP in bone regeneration after cyst enucleation in pediatric patients - A clinical study. Journal of clinical pediatric dentistry. 2010; 35(1): 81-87. 
7. Marx R E, Carlson E R, Eichstaedt R M. Platelet rich plasma, growth factor enhancement for bone grafts. Oral Surg Oral Med Oral Pathol Oral Radiol Endod. 1998; 85: 638-646.

8. Kanno T, Takahas T, Tsujisawa T. Platelet-Rich Plasma Enhances Human Osteoblast-Like Cell Proliferation and Differentiation. J Oral Maxillofac Surg. 2005; 63:362-369.

9. Bielecka AC, Bielecki T, Gazdik TS. Improved treatment of mandibular odontogenic cysts with platelet rich gel. Oral Surg Oral Med Oral Pathol Oral Radiol Endod. 2008; 105: 423-429.

10. Oyama T, Nishimoto S, Tsugawa T. Efficacy of Platelet-Rich Plasma in Alveolar Bone Grafting. J Oral Maxillofac Surg. 2004; 62:555-558.

11. Sammartino G, Tia M, Marenzi G. Use of Autologous Platelet-Rich Plasma (PRP) in Periodontal Defect Treatment After Extraction of Impacted Mandibular Third Molars. J Oral Maxillofac Surg. 2005; 63:766-770.

12. 47

13. Dohan DM, Choukroun J, Diss A, Dohan SL. Platelet-rich fibrin (PRF): A second-generation platelet concentrate.Part II: Platelet-related biologic features. Oral SurgOral Med Oral Pathol Oral Radiol Endod. 2006; 101: E45-50.

14. Lee JW, Kim SG, Kim YJ. Restoration of a peri-implant defect by platelet-rich fibrin. Oral Surg Oral Med Oral Pathol Oral Radiol. 2012; 113:459-463.

15. Dohan DM, Choukroun J, Diss A, Dohan SL. Platelet-rich fibrin (PRF): A second-generation platelet concentrate.Part III: Leucocyte activation: A new feature for platelet concentrates? Oral Surg Oral Med Oral Pathol Oral Radiol Endod. 2006; 101: E51-5.

16. Choukroun J, Diss A, Simonpieri A, Girard MO. Platelet-rich fibrin (PRF): A second-generation platelet concentrate.Part V: Histologic evaluations of PRF effects on bone allograft maturation in sinus lift.Oral Surg Oral Med Oral Pathol Oral RadiolEndod. 2006; 101:299-303.

17. Choukroun J, Diss A, Simonpieri A, Girard MO. Platelet-rich fibrin (PRF): A second-generation platelet concentrate.Part V: Histologic evaluations of PRF effects on bone allograft maturation in sinus lift.Oral Surg Oral Med Oral Pathol Oral RadiolEndod. 2006; 101:299-303.

18. Antoine Diss, Dohan DM, Mouhyi J. Osteotome sinus floor elevation using Choukroun's platelet-rich fibrin as grafting material: a 1-year prospective pilotstudy with microthreaded implants. Oral Surg Oral Med Oral Pathol Oral Radiol Endod .2008; 105: 572-579.

19. Pradeep A R, Rao NS, Agarwal E. Comparative evaluation of autologous PRF and PRP in the treatment of 3 wall intra bony defects in chronic periodontitis: A randomized controlled clinical trial. J Periodontol. 2012. 